Horizontes. Revista de Investigación en Ciencias de la Educación

ISSN 2616-7964

julio-septiembre, 2017

HORIZONTES

Volumen 1, Número 3

www.revistahorizontes.org

pp. 33-41

\title{
Los inicios de la república y el proceso pedagógico decimonónico (1830-1870)
}

\section{The beginnings of the republic and the nineteenth-century pedagogical process (1830-1870)}

Betsy Zabala Morales

betzabala_m81@hotmail.com

Universidad Militar Bolivariana de Venezuela

Recibido: 13-10-2016 / Revisado: 18-10-2016 / Aceptado: 20-11-2016 / Publicado: 10-07-2017

RESUMEN

La educación es en sí misma un proceso dinámico, por lo que presenta cambios permanentes y continuos en toda su estructura. De esta manera, se puede afirmar que la educación desde cualquier modalidad, procura generar un aprendizaje a través de la interacción del ser humano con el medio ambiente; convirtiéndose entonces, en el resultado de la experiencia. El medio ambiente estará determinado por un momento histórico determinado, que rige una sociedad y una estructura política acorde con el mismo. De allí que sea la historia la encargada de describir ese momento de la vida del ser humano, entendiendo el porqué de los hechos; es decir, las causas y las consecuencias de los mismos. En el caso de Venezuela, la época entre 1830 y 1870 fue un periodo de reconstrucción de la República; siendo la educación la herramienta fundamental de desarrollo para el país. La elaboración de este artículo se ha realizado mediante la revisión documental de aportes realizados por diversos autores en torno a la época de los inicios de la república entre 1830 y 1870; desarrollándose de esta manera una investigación de carácter argumentativo a través de categorías de estudio. La metodología de trabajo, se desarrolla en base al análisis crítico. El artículo se organiza en cuatro partes: en primer lugar, la introducción, donde se da un esbozo general de la investigación; en segundo lugar, se muestra un contexto general de la situación país entre los años 1830 y 1870; en tercer lugar, se desarrolla una evidencia crítica de la evolución de la educación en estos años. Seguidamente, en cuarto lugar, se presenta un esbozo de las aproximaciones conceptuales realizadas por Mirla Alcibíades en su libro "La heroica aventura de construir una república: 1830-1865" a manera de reflexión. El estudio permite concluir que el proceso de construcción del sistema escolar que se inicia formalmente en Venezuela en el período de la Gran Colombia, forma parte del surgimiento y desarrollo de los sistemas nacionales de instrucción pública en el ámbito iberoamericano; manteniéndose en constantes cambios y desarrollo para la formación de la sociedad.

Palabras clave: Inicios de la república, Educación entre 1830-1870, Decreto instrucción pública gratuita y obligatoria, Código de instrucción, Colegios nacionales 


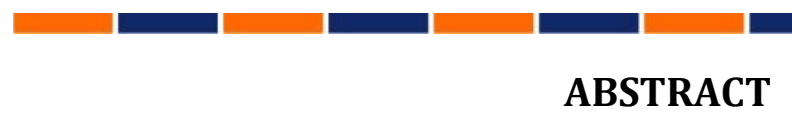

Education is in itself a dynamic process, so it presents permanent and continuous changes throughout its structure. In this way, it can be affirmed that education from any modality, seeks to generate learning through the interaction of the human being with the environment; becoming, then, the result of experience. The environment will be determined by a specific historical moment, which governs a society and a political structure in accordance with it. Hence, history is responsible for describing that moment in the life of the human being, understanding the reason for the events; that is, the causes and consequences of them. In the case of Venezuela, the period between 1830 and 1870 was a period of reconstruction of the Republic; being education the fundamental tool of development for the country. The elaboration of this article has been made through the documentary review of contributions made by various authors around the time of the beginnings of the republic between 1830 and 1870; In this way, an investigation of argumentative character is developed through study categories. The work methodology is developed based on critical analysis. The article is organized in four

«No se puede separar la educación de la cultura porque sin instrucción es imposible que haya cultura, desde luego la educación es una faceta del proceso cultural»

\section{María Alcibíades}

\section{INTRODUCCIÓN}

La educación es en sí misma un proceso dinámico, por lo que presenta cambios permanentes y continuos en toda su estructura; sin embargo, ello no implica que esto ocurra en su propia esencia, la cual se traduce en preparar al individuo en el presente para su desarrollo en el futuro. De esta manera, se puede afirmar que la educación desde cualquier modalidad, procura generar un aprendizaje a través de la interacción del ser humano con el medio ambiente; convirtiéndose entonces, en el resultado de la experiencia. parts: first, the introduction, where a general outline of the research is given; Secondly, it shows a general context of the country situation between the years 1830 and 1870; third, a critical evidence of the evolution of education in these years is developed. Then, in fourth place, an outline of the conceptual approaches made by Mirla Alcibíades in her book "The heroic adventure of building a republic: 18301865 " as a reflection is presented. The study allows concluding that the construction process of the school system that formally begins in Venezuela in the period of the Gran Colombia is part of the emergence and development of national systems of public instruction in the Ibero-American sphere; keeping in constant changes and development for the formation of society.

Key words: Beginnings of the republic, Education between 1830-1870, Decree of free and obligatory public instruction, code of instruction, national schools

El medio ambiente estará determinado por un momento histórico determinado, que rige una sociedad y una estructura política acorde con el mismo. De allí que sea la historia la encargada de describir ese momento de la vida del ser humano, entendiendo el porqué de los hechos; es decir, las causas y las consecuencias de los mismos. En el caso de Venezuela, la época entre 1830 y 1870 fue un periodo de reconstrucción de la República; siendo la educación la herramienta fundamental de desarrollo para el país.

La elaboración de este artículo se ha realizado mediante la revisión documental de aportes realizados por diversos autores en torno a la época de los inicios de la república entre 1830 y 1870; desarrollándose de esta manera una investigación de carácter argumentativo a través de categorías de estudio. La metodología de trabajo, se desarrolla en base al análisis crítico. 
El artículo se organiza en tres partes: en primer lugar, se muestra un contexto general de la situación país entre los años 1830 y 1870; en segundo lugar se desarrolla una evidencia crítica de la evolución de la educación en estos años, ello a través de cuatro (4) grandes apartados: (a) Cronología educativa desde 1830 hasta 1870, (b) El Código de Instrucción Pública de 1843 y su influencia en la Educación Primaria, (c) Los Colegios Nacionales de la mano con el desarrollo de la Educación Superior; y (d) el Decreto de Instrucción Pública, Gratuita y Obligatoria de 1870. Seguidamente, en tercer lugar, se presenta un esbozo de las aproximaciones conceptuales realizadas por Mirla Alcibíades en su libro "La heroica aventura de construir una república: 18301865" a manera de reflexión y/o conclusión; finalmente, se presentan las referencias bibliográficas que sustentan este artículo.

\section{Contexto general de la situación país entre los años 1830 y 1870}

Entre los años de 1830 hasta 1870, Venezuela se encontraba en un proceso de reconstrucción de la República, buscando instaurar el orden liberal, el cual se encontraba de boga en Estados Unidos e Inglaterra. Para lograrlo, consideraban importante producir una revolución de las fuerzas productivas, romper con gran parte de los prejuicios y estructuras heredades del orden colonial, así como crear y consolidar un verdadero Estado Nacional, capaz de imponerse al Caudillismo.

El régimen del Caudillismo, se mantuvo por las Oligarquías, entendidas como gobiernos ejercidos exclusivamente por algunos grupos poderosos; los mismos se dividieron en dos: (a) la Oligarquía Conservadora y (b) la Oligarquía Liberal.
Siendo el principal caudillo de los conservadores el General José Antonio Páez y de los liberales el General José Tadeo Monagas.

La Oligarquía Conservadora mantenía un gobierno centro federalista, al cual habían accedido mediante la imposición y la dictadura; generando un exclusivismo político y económico, no permitiendo las reelecciones presidenciales. De allí que mantenían el sistema esclavista como parte de sus políticas de estado; no tenían el apoyo del clero, ni de los campesinos. Durante este período ejercieron sucesivamente la Presidencia de la República: José Antonio Páez (1830-1835); José María Vargas (1835-1836), cuyo mandato constitucional fue completado por el vicepresidente Andrés Narvarte (1836-1837), primero y, luego, por el vicepresidente Carlos Soublette (1837-1839); de nuevo José Antonio Páez (1839-1843) y Carlos Soublette (18431847).

La Oligarquía Liberal, por su parte, mantenía un gobierno autócrata llegando al poder tras ser elegidos. El gobierno estaba compuesto por hombres de todos los grupos y clases sociales; generaron la abolición de la esclavitud. Mantenían el apoyo de la mayoría de los venezolanos, pues se permitía la reelección del presidente. Durante este tiempo ocuparon la Presidencia de la República, José Tadeo Monagas (1848-1851), José Gregorio Monagas (1851-1855) y de nuevo José Tadeo Monagas (1855-1858).

Es importante acotar que durante estas dos etapas de la Oligarquía, el país vivió un caos económico muy fuerte, aunado a una guerra civil que contribuyó a la desorganización de la sociedad; trayendo como consecuencia una anarquía política, social, económica y educativa, y con ello una 
sociedad donde la mayoría de sus integrantes no sabían ni leer ni escribir.

\section{Evidencia Crítica de la Evolución de la Educación en Venezuela desde 1830 hasta 1870}

\section{Cronología educativa desde 1830 hasta 1870}

En esta etapa de la historia educativa de Venezuela y de la mano del liberalismo decimonónico, se procura el desarrollo de una escuela en el tiempo de la República bajo las ideas de la Ilustración. En el entendido de que ese pensamiento pedagógico ilustrado, se encontraba orientado a elevar el nivel cultural de las sociedades; buscando una realidad científica y la liberación del pensamiento a través de la razón. Las características principales de este pensamiento giraban en torno a la naturaleza, la razón, la igualdad y la libertad.

De acuerdo a Fernández (1981), aun cuando la concepción educativa de la época partía de la idea de una sociedad de igualdades sociales y políticas, no fue sino hasta el año de 1854 cuando se promulgó la liberación de los esclavos. Resaltando que el sistema político manipulaba la Constitución para hacer de ella un traje a la medida; adaptado a sus necesidades y exigencias en materia política, económica y social.

Los intelectuales de la época observaban la educación desde dos perspectivas: (1) La educación como un medio para la creación de los ciudadanos republicanos, con grados académicos (profesiones) o sin estos (artes y oficios); y (2) La educación como la vía más importante para el progreso del país, a través de un proceso socializador.

Considerando estas perspectivas, el hecho educativo entre 1830 y 1870 puede desarrollarse en la siguiente cronología:

1. El Congreso de 1830 dispuso que la enseñanza primaria fuera desarrollada por cada Entidad Provincial. Sin embargo, como consecuencia de los embates de la separación de la Gran Colombia, al gobierno le resultaba difícil obtener los fondos que eran necesarios para la educación. Ello trajo como consecuencia que para el año de 1830 el país contaba con tan solo cien (100) escuelas primarias municipales.

2. Para el año de 1831, el Secretario de Interior y Justica Antonio Leocadio Guzmán anuncia la creación de los Colegios Nacionales; procurando con ello corregir el vicio de la promiscuidad de estudios, tales como colegios ofreciendo carreras universitarias y al mismo tiempo estudios de la primera enseñanza. En ese mismo año, es abierta la Escuela Inglesa de la señora Campbell, para la educación de niñas.

3. En 1833, el Presidente Páez estableció por decreto el funcionamiento de la Biblioteca Nacional.

4. Para 1834, funcionan 7 Colegios Nacionales: El Tocuyo, Barquisimeto, Carabobo, Margarita, Coro, Trujillo y Guanare.

5. En 1837, abre el Colegio de las Educandas de las señoras Encarnación, Teresa y Concepción Luque; y el Colegio de la Concepción de las hermanas Dolores y Manuela Guido. En 1838, se crea la Nueva Escuela de Señoritas. Sin embargo, es hasta 1840, que se decreta el funcionamiento del plantel oficial Colegio Nacional de Niñas.

6. El mensaje del Dr. José María Vargas en el año de 1836, dedicó gran atención al tema educativo. Es importante recordar que el país continuaba bajo la legislación del Congreso de Cúcuta y del Ejecutivo Gran Colombiano. Razón por la cual se crea en 1838, la Dirección General de Instrucción Pública según decreto del Presidente Carlos Soublette. La Dirección General de Instrucción Pública se crea como una instancia ejecutiva adscrita a la Secretaria de Interior y Justica, para llevar las estadísticas en materia educativa en el país. De acuerdo a Rojas (s/f), con la creación de esta Dirección, el Estado Docente da sus primeros pasos como promotor, financiador, evaluador y supervisor del proceso educativo. (Ver Imagen 2). 
Imagen 2. Memorias de la Dirección General de Instrucción Pública (1839-1852)

\begin{tabular}{|c|c|c|}
\hline AÑO & $\begin{array}{c}\text { CANTIDAD DE ESCUELAS } \\
\text { (PÚBLICASY PRIVADAS) }\end{array}$ & $\begin{array}{c}\text { POBLACIÓN } \\
\text { (niños de ambos sexos) }\end{array}$ \\
\hline 1839 & 121 & No se especifica \\
\hline 1840 & 216 & 7.945 estudiantes \\
\hline 1845 & 428 & 12.905 estudiantes \\
\hline 1852 & 341 & 10.216 estudiantes \\
\hline
\end{tabular}

Fuente: Fernández H., R. (1981) Memorias de cien años. Ediciones del Ministerio de Educación

7. Para 1855, el Ministro de Interior y Justicia, Simón Planas, ordena el régimen educativo en 3 niveles, claramente definidos en sus objetivos y con planteles: primario, secundario y superior.

Solicitando a su vez, realizar las pruebas de selección para el ingreso a las universidades.

8. El 27 de junio de 1870, el Presidente Antonio Guzmán Blanco promulga el Decreto de Instrucción Pública Gratuita y Obligatoria, dando paso a un desarrollo a nivel educativo en el país.

\section{El Código de Instrucción Pública de 1843 y su influencia en la Educación Primaria}

La concepción educativa dentro del orden liberal, movida por las ideas de la Ilustración, cuyo pensamiento emprendía un camino hacia la instauración de una sociedad libre e igualitaria; da paso a la promulgación por el Congreso venezolano del Código de Instrucción Pública de 1843 conformado por catorce (14) leyes para reglamentar la educación primaria, secundaria y superior. Aprobándose así el primer reglamento de enseñanza en Venezuela.

Considerando a Rojas (s/f) es sobre el entramado jurídico que se inicia la construcción del sistema escolar público de
Venezuela, con sus virtudes, debilidades, ausencias y defectos.

A pesar de dar paso a la reglamentación en materia educativa en el país, este Código fue, de acuerdo a Fernández (1998), fundamentalmente una legislación para la educación universitaria, pues en materia de educación primaria continúa primando el principio centro- federalista. Las leyes que conforman este Código sufren reformas sucesivas, que obedecían en la generalidad de los casos a motivaciones casuales. Esta legislación es derogada por el gobierno del Presidente Antonio Guzmán Blanco en la segunda parte del siglo XIX.

En tanto ello es así, se pone de manifiesto que hacia el año de 1852, el estado de la educación primaria era lastimoso, las provincias no tenían rentas para fomentarla. El currículo educativo de educación primaria se centraba en la memorización de contenidos que los docentes leían a sus estudiantes; aun cuando se procuraba la enseñanza de la lectura, la escritura, la aritmética, urbanidad, moral y religión.

Destacándose que entre el año de 1850 y 1870 no existió ningún esfuerzo que representara un impulso significativo para la educación de este nivel; razón por la cual el índice analfabetismo en Venezuela era considerablemente elevado. 


\section{Los Colegios Nacionales de la mano con el desarrollo de la Educación Superior}

En el año de 1833, se inician bajo la dirección de Juan Manuel Cajigal los Colegios Nacionales; ello con el fin de suministrar la enseñanza científica. Se dictaba cátedras de gramática, castellano, filosofía y latín; en los diferentes cursos de Medicina, Derecho y
Filosofía; otorgando al culminar la enseñanza títulos de Bachiller para poder ingresar a la Universidad.

Los planteles inician actividades a medida que aseguran sus rentas y la disponibilidad de estudiantes y de personal docente. Se abren sucesivamente doce (12) colegios, tal como se muestra en la Tabla 1.

Tabla 1. Primeros Colegios Nacionales.

\begin{tabular}{c|c}
\hline \multicolumn{2}{c}{ Primeros Colegios } \\
\hline Carabobo, 11 de agosto de 1833 & Margarita, 1835 \\
\hline Cumaná, 28 de febrero de 1834 & Trujillo, 1835 \\
\hline Coro, 26 de noviembre de 1833 & El Tocuyo, 1 de mayo de 1835 \\
\hline Guayana, 8 de abril de 1834 & Calabozo, 1839 \\
\hline Guanare, 28 de febrero de 1834 & Maracaibo, 2 de marzo de 1837 \\
\hline Barquisimeto, 20 de enero de 1835 & Barcelona, 1842 \\
\hline
\end{tabular}

Fuente: Herrera G., L. (2008) Los Colegios Nacionales. Revista de la Sociedad Venezolana de Historia de la Medicina

Estos colegios eran autónomos, por lo cual los dirigía un rector, un vicerrector y un consejo docente. En algunos de estos colegios, existía paralelamente una escuela de primeras letras. En el año de 1839, el General Páez aprobó el Decreto Orgánico de los Colegios Nacionales, perfeccionado por el Código de Instrucción Pública; el mismo constaba de 15 leyes.
De acuerdo a Herrera (2008), en la Memoria al Congreso de 1845 el Dr. Vargas insiste en la escasez de planteles (ver imagen 3), de falta de información sobre su funcionamiento y de personal calificado para el mismo. Tal aseveración permite destacar, que la falta de estudiantes o de directores competentes y las dificultades económicas ocasionó el cierre o decaimiento de algunos de estos colegios. 


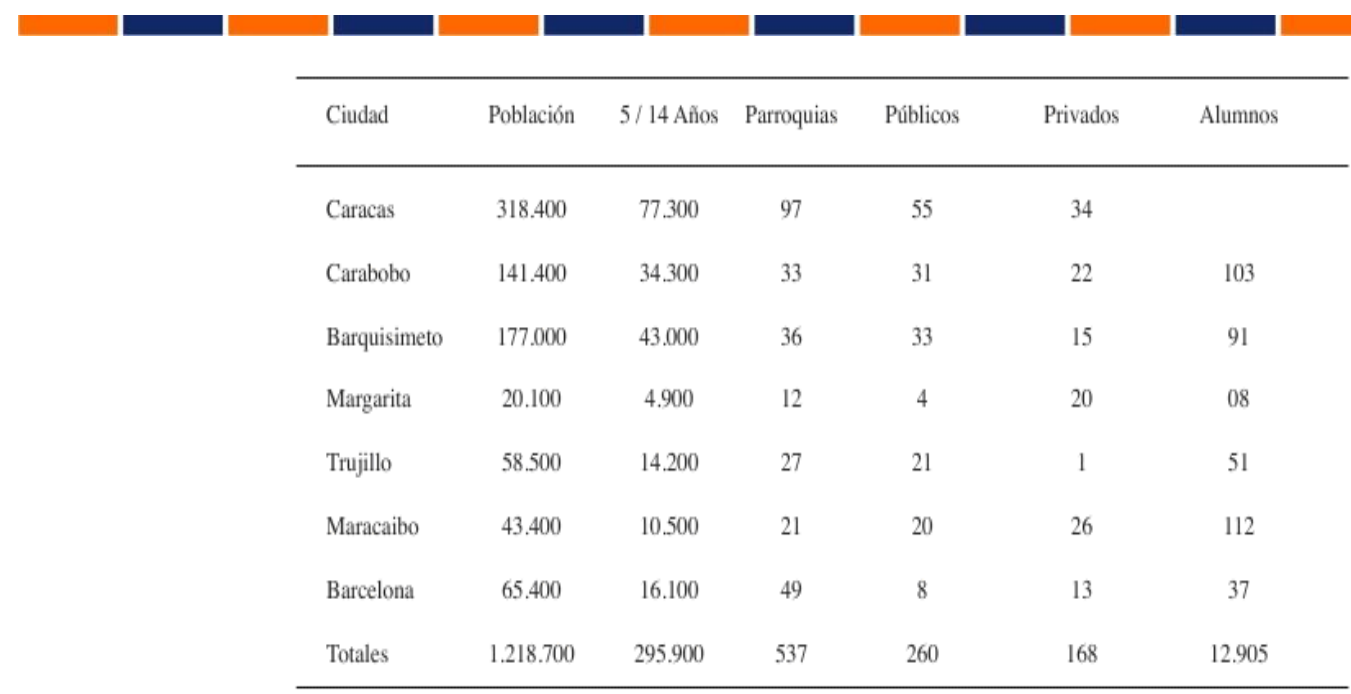

Imagen 3. Memoria del Dr. Vargas al Congreso de 1845.

Fuente: Herrera G., L. 2008) Los Colegios Nacionales. Revista de la Sociedad Venezolana de Historia de la Medicina

En el marco de estos colegios nacionales, en Caracas funcionaron colegios privados, de renombre, dirigidos por hombres competentes como Feliciano Montenegro y Colón, José Ignacio Paz Castillo, Juan José Aguerrevere, Tomás Víctor Bermúdez y Juan Vicente González. Allí se familiarizó la juventud venezolana con la historia nacional y las letras extranjeras; así como con ciencias experimen-tales, físicas y naturales, dirigidas por Juan Manuel Cajigal y por Fermín Toro.

La educación universitaria, como escalón consecutivo de los colegios nacionales, quedaba adscrita a los poderes nacionales. Durante los años de 1830 a 1870, las universidades sufrieron algunas transformaciones. Por su parte, la Universidad de Caracas (Universidad Central desde 1875) abrió sus puertas en 1725 unida al seminario de Santa Rosa de Lima. En 1827, fue colocada por Simón Bolívar bajo la dirección del Dr. José María Vargas. Este introdujo aparatos de física y química; colecciones de ciencias naturales y le da a la enseñanza una orientación experimental. Funda la cátedra de anatomía y la de cirugía. Por otro lado, la Universidad de Mérida (Universidad de los Andes desde 1875), es fundada en 1810. Para el año de 1832, bajo la dirección del presbítero doctor
Ignacio Fernández de Peña inicia una lenta labor de transformación.

Es importante destacar que en el período que va desde 1838 a 1848, la Universidad de Caracas confiere grados de Doctor o Licenciado en Derecho, Medicina y Teología; y doce grados de Maestro en Filosofía. En cambio, la Universidad de Mérida, para el mismo período, solo gradúa 27 Doctores o Licenciados y 16 Maestros de Filosofía.

\section{El Decreto de Instrucción Pública, Gratuita y Obligatoria de 1870}

El Presidente General Antonio Guzmán Blanco decreta el 27 de junio de 1870, el Decreto de Instrucción Pública, Gratuita y Obligatoria con la finalidad de impulsar la educación básica, dirigida especialmente a la población carente de recurso; procurando con ello la disminución del analfabetismo en el país.

El decreto establece la obligatoriedad de la enseñanza gratuita de lo contentivo a principios generales de aritmética, idioma castellano, moral ciudadana y fundamentos de la Constitución Federal. Obligando de esta manera a los padres y /o tutores a instruir a sus hijos en las señaladas temáticas, o a contratar un maestro para el mismo cometido. 
De la mano de este decreto, se crea una Dirección Nacional de Instrucción Primaria con juntas seccionales en los estados; y un impuesto especial para la dotación de material de las escuelas que se crearían en el futuro. Asimismo, se fortalecieron los Colegio Nacionales, ahora llamados Federales; para la enseñanza de la educación media.

\section{Para reflexionar...}

La historia de la educación en Venezuela, comprende un sinfin de hechos que quizás en ocasiones solo presentan una cara de la moneda; y dejan al lector a la expectativa de más información. En su libro La heroica aventura de construir una república 18301865, Mirla Alcibíades (2004) realiza un esbozo de aquellos aspectos que parecieron olvidados en la historia educativa que se ha desarrollado a lo largo de esta investigación.

Dice Alcibíades (2004) que la moral constituía un requerimiento común en todos y cada uno de los discursos de la vida; de allí que "El verdadero reto surgió cuando se quiso vincular la moral con la vida pública, con lo social" (p.59), por ello los "discursos que hablaban de una escolaridad para la ciudadanía, de la moral ciudadana, fueron entusiastas"(p.95). La educación, aun cuando era una responsabilidad del Estado en materia de estructura y de contenidos académicos; sería la familia el pilar que sostuviese la formación de la moral y de la integración a la sociedad; de allí que “...el papel que le correspondía desempeñar a la familia en el proceso de la formación nacional...con la importancia de otorgarle al niño/niña el correspondiente lugar en el entramado social" (p. XIII).

1. Otro hecho importante que refleja Alcibíades (2004) es el desarrollo de la Literatura durante este período; un período en el cual el Romanticismo se encontraba en boga a nivel mundial. Sin embargo, para Venezuela se considera que este fue el "tiempos del romanticismo tardío en nuestra literatura". Entre los hechos que se destacaron están:

2. En 1839, se promulga la ley de imprenta. Se publica el «Arte de hablar en prosa y verso» de José Gómez Hermosilla.

3. 1842, es el año del establecimiento del Gabinete de Lectura de la librería Caballerizo. Ya que no existía aun una biblioteca pública se pensó que sería una manera de sustituirla.

4. La prensa periódica comienza a conceder mayor espacio a la producción literaria nacional (poesía)

5. En 1856, procede un gran ensayo: Cosas sabidas y cosas por saberse, de Cecilio Acosta(1818-1881); allí a través de la bella prosa se tratan graves problemas nacionales.

6. En 1865 fue creada la literatura infantil con El libro de la infancia de don Amenodoro Urdaneta; circuló también ese año el único tomo de la Revista Literaria de Juan Vicente González, allí se encontraba la mayor obra de la prosa romántica venezolana: su Biografía de José Félix Ribas y se imprimió, en Nueva York, la Vida de Bolívar de don Felipe Larrazábal (1816-1873), la primera gran biografía del Libertador, uno de los libros más famosos del siglo XIX

Finalmente, en términos generales, se concluye que el proceso de construcción del sistema escolar que se inicia formalmente en Venezuela en el período de la Gran Colombia, forma parte del surgimiento y desarrollo de los sistemas nacionales de instrucción pública en el ámbito iberoamericano. Siendo un modelo educativo que continua en constantes cambios y desarrollo para la formación de la sociedad; lo cual se evidencia al realizar una 
revisión cronológica del continuo educativo en

Venezuela; tema de envergadura para próximas investigaciones.

\section{REFERENCIAS}

Alcibíades, Mirla (2004) La heroica aventura de construir una república 1830-1865. Caracas: Monte Ávila Editores. Extraído del taller crítico de Lovera De-Sola, R. (2012)

Brito Figueroa, F. (1978). Historia económica y social de Venezuela. Tomo II. Ediciones de la Biblioteca. Universidad Central de Venezuela. Caracas.

Decreto de Instrucción Pública Gratuita y Obligatoria en Venezuela de 1870. Consultado el 24 de septiembre de 2016. Disponible en: http://www.iconosdevenezuela.com/?p=2 1328

Fernández H., R. (1998) La educación en el siglo XIX. Biblioteca Digital Andina. Consultado el 12 de agosto de 2016, Disponible en http://www.comunidadandina.org/BDA/d ocs/VE-EDU-0011.pdf

Fernández H., R. (1981) Memorias de cien años. Ediciones del Ministerio de Educación T. II P. 5.

Herrera G., L. (2008) Los colegios nacionales. Revista de la Sociedad 57, № 1-2.

Disponible en http://revista.svhm.org.ve/ediciones/2008 /1-2

Rojas, R. (s/f). Ensayo: Educación y nación: La formación del sistema escolar público en Venezuela. Realizado en el $70^{\circ}$ aniversario del Instituto Pedagógico de Caracas. Consultado el 12 de agosto de 2016. Disponible en http://www.saber.ula.ve/bitstream/12345 6789/21085/2/articulo9.pdf

Saavedra, Luis B. (s/f). Ensayo sobre historia de la educación en Venezuela. Caracas. Consultado el 12 de agosto de 2016. Disponible en http://www.uft.edu.ve/postgrado/omega digital/OD_marzo2006/descargables/ensa yo_historia\%20de $\% 20$ la $\% 20$ educacion $\% 2$ 0en\%20venezuela.pdf

Introducción, Capítulo Primero. www.sociedadheidegger.org. Consulta mayo 302016

Villarreal Hernández Jesús A. (2014. Episteme teorético desde el Ser de la docencia universitaria. Valencia, Venezuela: ARJÉ Revista de Postgrado FACE-UC. 8 (14) Enero-Junio, 2014. Edición Especial / 247270

Waldenfels, Bernhard (1997). De Husserl a Derrida. Introducción a la fenomenología. Barcelona, España: Paidós, pp. 59-62 